\title{
Unitary Quantum Physics with Time-Space Noncommutativity
}

\author{
A. P. Balachandran \\ Physics Department, Syracuse University \\ Syracuse, NY, 13244-1130, USA \\ E-mail: bal@phy.syr.edu

\section{T. R. Govindarajan \\ The Institute of Mathematical Sciences \\ C. I. T. Campus Taramani, Chennai 600 113, India \\ E-mail: trg@imsc.res.in}

\section{Molina and P. Teotonio-Sobrinho \\ Instituto de Física, Universidade de São Paulo \\ C.P. 66318, São Paulo, SP, 05315-970, Brazil \\ E-mail: cmolina@fma.if.usp.br, teotonio@fma.if.usp.br}

\begin{abstract}
In this work quantum physics in noncommutative spacetime is developed. It is based on the work of Doplicher et al. which allows for time-space noncommutativity. The Moyal plane is treated in detail. In the context of noncommutative quantum mechanics, some important points are explored, such as the formal construction of the theory, symmetries, causality, simultaneity and observables. The dynamics generated by a noncommutative Schrödinger equation is studied. We prove in particular the following: suppose the Hamiltonian $H$ of a quantum mechanical particle on spacetime $\mathbb{R}^{N-1} \times \mathbb{R}$ has no explicit time dependence, and the spatial coordinates commute in its noncommutative form (the only noncommutativity being between time and a space coordinate). Then the noncommutative version $\hat{H}$ of $H$ and $H$ have identical spectra.
\end{abstract}

Keywords: Field Theories in Lower Dimensions, Non-Commutative Geometry. 


\section{Contents}

1. Introduction 2

2. Qualitative Remarks 2

2.1 Symmetries 2

2.1.1 Translations 2

2.1.2 The Lorentz Group 3

2.1.3 P, T, C Symmetries

2.1.4 Further Automorphisms

2.2 Causality 1

2.3 The Spin-Statistics Connection

3. Representation Theory 5

3.1 The Commutative Case 5

3.1.1 The Positive Map 5

3.1 .2 The Null Space $\mathcal{N}_{t}^{0}$

3.1.3 The Quantum Mechanical Hilbert Space $\mathcal{H}_{t}^{0}$

3.1.4 On Observables 7

3.1.5 Time-dependent $H$

3.1.6 Is Time an Observable? 8

3.1.7 On the Time-dependence of $\mathcal{H}_{t}^{0}$

3.2 The Noncommutative Case 9

3.2.1 The Inner Product 9

3.2.2 The Schrödinger Constraint 10

3.2.3 Remarks on Time for $\theta \neq 0$

3.2.4 Time-dependence for $\theta=0 \Longrightarrow$ Spatial nonlocality for $\theta \neq 0$

3.2 .5 Observables 12

3.2.6 The Scalar Products $(., .)_{S}$ and $(., .)_{V}$

4. Examples 13

4.1 Plane Waves 14

4.2 A Spectral Map 14

5. Conserved Current 15

6. Towards Quantum Field Theory 15 


\section{Introduction}

Considerations based on quantum gravity and black hole physics led to the suggestion several years ago [1] that spacetime commutativity may be lost at the smallest scale, the commutators of time and space coordinates $\left(\hat{x}_{0}\right.$ and $\left.\hat{x}_{i}\right)$ having the form

$$
\left[\hat{x}_{\mu}, \hat{x}_{\nu}\right]=i \theta_{\mu \nu} \mathbb{I},
$$

with $\theta_{\mu \nu}$ being constants of the order of the square of Planck length. String theory also incorporates relations like (1.1).

Commutators such as (1.1) actually have a much more ancient origin. They seem to have first appeared in a letter from Heisenberg to Peierls in 1930 [2]. Spacetime noncommutativity was later revived by Snyder [3] who sought to use it to regularize quantum field theories (qft's), and then by Yang [4]. Madore [5] also attributes similar ideas to Dirac. Among the early works in noncommutative spacetime is that of Kempf et al. [6]. A subsequent related work is that of Lizzi et al. [7].

Conventional studies of (1.1) assume that $\theta_{0 i}=0$ so that the time coordinate commutes with the rest. There are even claims that qft's based on (1.1) are nonunitary if $\theta_{0 i} \neq 0$.

In contrast, in a series of fundamental papers, Doplicher et al. [1] have studied (1.1) in complete generality, without assuming that $\theta_{0 i} \neq 0$ and developed unitary qft's which are ultraviolet finite to all orders.

This paper is based on the work of Doplicher et al. Using their ideas, we systematically develop unitary quantum mechanics based on (1.1). It indicates where to look for phenomenological consequences of (1.1) and also easily leads to the considerations of Doplicher et al. [1] on qft's.

The relation (1.1) will be treated with $\theta$ being constant. Our focus is on time and its noncommutativity with spatial coordinates. For this purpose, it is enough to examine (1.1) on a $(1+1)$-spacetime and replace it by

$$
\left[\hat{x}_{\mu}, \hat{x}_{\nu}\right]=i \theta \varepsilon_{\mu \nu} \mathbb{I}, \varepsilon_{\mu \nu}=-\varepsilon_{\nu \mu}, \varepsilon_{01}=1 \text {. }
$$

We assume with no loss of generality that $\theta>0$, as we can change its sign by flipping $\hat{x}_{1}$ to $-\hat{x}_{1}$. We denote by $\mathcal{A}_{\theta}\left(\mathbb{R}^{2}\right)$ the unital algebra generated by $\hat{x}_{0}, \hat{x}_{1}$ and $\mathbb{I}$.

\section{Qualitative Remarks}

\subsection{Symmetries}

If a group of transformations cannot be implemented on the algebra $\mathcal{A}_{\theta}\left(\mathbb{R}^{2}\right)$ generated by $\hat{x}_{\mu}$ with relation (1.1), then it is not likely to be a symmetry of any physical system based on (1.1) [8]. So let us check what are the automorphisms of (1.1).

\subsubsection{Translations}

First we readily see that spacetime translations $\mathcal{U}(\vec{a}), \vec{a}=\left(a_{0}, a_{1}\right), a_{\mu} \in \mathbb{R}$, are automorphisms of $\mathcal{A}_{\theta}\left(\mathbb{R}^{2}\right)$ : with

$$
\mathcal{U}(\vec{a}) \hat{x}_{\mu}=\hat{x}_{\mu}+a_{\mu},
$$


we see that

$$
\left[\mathcal{U}(\vec{a}) \hat{x}_{\mu}, \mathcal{U}(\vec{a}) \hat{x}_{\nu}\right]=i \theta \varepsilon_{\mu \nu} .
$$

The existence of these automorphisms allows the possibility of energy-momentum conservation. The time-translation automorphism

$$
U(\tau):=\mathcal{U}((\tau, 0))
$$

is of particular importance. Without it, we cannot formulate conventional quantum physics.

The infinitesimal generators of $\mathcal{U}(\vec{a})$ can be defined by writing

$$
\mathcal{U}(\vec{a})=e^{-i a_{0} \hat{P}_{0}+i a_{1} \hat{P}_{1}} .
$$

Then we have

$$
\hat{P}_{0}=-\frac{1}{\theta} \operatorname{ad} \hat{x}_{1}, \hat{P}_{1}=-\frac{1}{\theta} \operatorname{ad} \hat{x}_{0}, \operatorname{ad} \hat{x}_{\mu} \hat{a} \equiv\left[\hat{x}_{\mu}, \hat{a}\right], \hat{a} \in \mathcal{A}_{\theta}\left(\mathbb{R}^{2}\right) .
$$

The relations (2.5) show that the automorphisms $\mathcal{U}(\vec{a})$ are inner.

\subsubsection{The Lorentz Group}

It is a special feature of two dimensions that the $(2+1)$ connected Lorentz group is an inner automorphism group of (1.1). The above group is the two-dimensional projective symplectic group, the symplectic group quotiented by its center $\mathbb{Z}_{2}$. Its generators are $\operatorname{ad} \hat{J}_{3}$ and $\operatorname{ad} \hat{K}_{a}$, where

$$
\hat{J}_{3}=\frac{1}{4 \theta}\left(\hat{x}_{0}^{2}+\hat{x}_{1}^{2}\right), \hat{K}_{1}=\frac{1}{4 \theta}\left(\hat{x}_{0} \hat{x}_{1}+\hat{x}_{1} \hat{x}_{0}\right), \hat{K}_{2}=\frac{1}{4 \theta}\left(\hat{x}_{0}^{2}-\hat{x}_{1}^{2}\right),
$$

with the ad notation explained by (2.5). Although this group generates inner automorphisms, it cannot be implemented on the quantum Hilbert space because, as we shall later see, $\hat{x}_{0}$ is not an operator on the physical Hilbert space.

The algebra $\mathcal{A}_{\theta}\left(\mathbb{R}^{2}\right)$ is a *-algebra with

$$
\hat{x}_{\mu}^{*}=\hat{x}_{\mu} .
$$

We note that

$$
\hat{J}_{3}^{*}=\hat{J}_{3}, \hat{K}_{a}^{*}=\hat{K}_{a}
$$

\subsubsection{P, T, C Symmetries}

There are certain important transformations which are automorphisms for $\theta=0$, but not for $\theta \neq 0$. One such is parity $P$ :

$$
P: \hat{x}_{0} \rightarrow \hat{x}_{0}, \hat{x}_{1} \rightarrow-\hat{x}_{1}, \mathbb{I} \rightarrow \mathbb{I}
$$

We want it furthermore to be linear. But that does not preserve (1.1) if $\theta \neq 0$ :

$$
P:\left[\hat{x}_{0}, \hat{x}_{1}\right] \rightarrow-\left[\hat{x}_{0}, \hat{x}_{1}\right], i \theta \mathbb{I} \rightarrow i \theta \mathbb{I} .
$$


In contrast, time-reversal $T$,

$$
T: \hat{x}_{0} \rightarrow-\hat{x}_{0}, \hat{x}_{1} \rightarrow \hat{x}_{1}
$$

is anti-linear,

$$
T: i \theta \mathbb{I} \rightarrow-i \theta \mathbb{I},
$$

so that it is an automorphism of $\mathcal{A}_{\theta}\left(\mathbb{R}^{2}\right)$.

Hence any theory based on (1.1) violates $P$ and $P T$. Superficially there seems to be no problem in writing charge conjugation invariant models based on (1.1). For such models, $C P T$ will also fail to be a symmetry [9].

The symmetries $P$ and $P T$ are automorphisms of the algebra which is the direct sum of $\mathcal{A}_{\theta}\left(\mathbb{R}^{2}\right)$ and $\mathcal{A}_{-\theta}\left(\mathbb{R}^{2}\right)$. In that case, spacetime will have two leaves. The Doplicher et al. models are based on such algebras.

\subsubsection{Further Automorphisms}

As fully discussed in [10, 11], infinitesimal transformations $\hat{x}_{\mu} \rightarrow \hat{x}_{\mu}+\delta \hat{x}_{\mu}$ of the form $\delta \hat{x}_{\mu}=\hat{f}_{\mu}\left(\hat{x}_{0}, \hat{x}_{1}\right)$ generate automorphisms of $\mathcal{A}_{\theta}\left(\mathbb{R}^{2}\right)$ if the condition

$$
\left[\hat{f}_{\mu}, \hat{x}_{\nu}\right]+\left[\hat{x}_{\mu}, \hat{f}_{\nu}\right]=0
$$

is satisfied. The associated group of transformations exhausts the noncommutative version of the area-preserving transformations (in two dimensions and connected to the identity), and includes the Lorentz group as a particular case.

\subsection{Causality}

It is impossible to localize (the representation of) "coordinate" time $\hat{x}_{0}$ in $\mathcal{A}_{\theta}\left(\mathbb{R}^{2}\right)$ sharply. Any state will have a spread in the spectrum of $\hat{x}_{0}$. This leads to failure of causality as explained by Chaichian et al. [12].

The following important point was emphasised to us by Doplicher [13]. In quantum mechanics, if $\hat{p}$ is momentum, $\exp (i \xi \hat{p})$ is spatial translation by amount $\xi$. This $\xi$ is not the eigenvalue of the position operator $\hat{x}$. In the same way, the amount $\tau$ of time translation in (2.3) is not "coordinate time", the eigenvalue of $\hat{x}_{0}$ [1]. It makes sense to talk about a state and its translate by $U(\tau)$. For $\theta=0$, it is possible to identify coordinate time with $\tau$ : the former is just a parameter we need for labelling time-slices of spacetime and increasing with $\tau$. But for $\theta \neq 0, \hat{x}_{0}$ is an operator not commuting with $\hat{x}_{1}$, and cannot be interchanged with $\tau$.

Concepts like duration of an experiment for $\theta=0$ [14] are expressed using $U(\tau)$. They carry over to the noncommutative case too.

\subsection{The Spin-Statistics Connection}

With loss of causality, one loses local qft's as well. As the best proofs of the spin-statistics connection require locality [15], we can anticipate the breakdown of this connection as well when $\mathcal{A}_{\theta}\left(\mathbb{R}^{2}\right)$ is generalised to $(3+1)$ dimensions. Precision experiments to test the spin-statistics connection are possible [16]. If signals for this violation due to $\theta \neq 0$ can be derived, good phenomenological bounds on $|\theta|$ should be possible. 


\section{Representation Theory}

Observables, states and dynamics of quantum theory are to be based on the algebra $\mathcal{A}_{\theta}\left(\mathbb{R}^{2}\right)$ defined by (1.1). The formalism for their construction, using the methods of the GNS approach [17] in the commutative and non-commutative contexts, will be explored in the following.

Now to each $\hat{\alpha} \in \mathcal{A}_{\theta}\left(\mathbb{R}^{2}\right)$, we can canonically associate its left and right regular representations $\hat{\alpha}^{L}$ and $\hat{\alpha}^{R}$,

$$
\hat{\alpha}^{L} \hat{\beta}=\hat{\alpha} \hat{\beta}, \hat{\alpha}^{R} \hat{\beta}=\hat{\beta} \hat{\alpha}, \hat{\beta} \in \mathcal{A}_{\theta}\left(\mathbb{R}^{2}\right),
$$

with $\hat{\alpha}^{L} \hat{\beta}^{L}=(\hat{\alpha} \hat{\beta})^{L}$ and $\hat{\alpha}^{R} \hat{\beta}^{R}=(\hat{\beta} \hat{\alpha})^{R}$. The carrier space of this representation is $\mathcal{A}_{\theta}\left(\mathbb{R}^{2}\right)$ itself.

But such representations are not enough for quantum physics. An "inner" product on $\mathcal{A}_{\theta}\left(\mathbb{R}^{2}\right)$ is needed for an eventual construction of a Hilbert space.

Doplicher et al. get this inner product using positive maps. Consider a map $\chi: \mathcal{A}_{\theta}\left(\mathbb{R}^{2}\right) \rightarrow \mathbb{C}$ with the usual properties of $\mathbb{C}$-linearity and preservation of $*: \chi\left(\hat{\alpha}^{*}\right)=\overline{\chi(\hat{\alpha})}$ (bar meaning complex conjugation). It is a positive map if

$$
\chi\left(\hat{\alpha}^{*} \hat{\alpha}\right) \geq 0 .
$$

Given such a map, we can set $\langle\hat{\alpha}, \hat{\beta}\rangle=\chi\left(\hat{\alpha}^{*} \hat{\beta}\right)$. It will be a scalar product if $\chi\left(\hat{\alpha}^{*} \alpha\right)=0$ implies $\hat{\alpha}=0$. If that is not the case, it is necessary to eliminate nonzero vectors of zero norm (null vectors).

We illustrate these ideas first in the context of the commutative case, when $\theta=0$. Then we generalise these ideas to (1.1) and in particular we discuss two positive maps. The first, due to Doplicher et al., is based on a symbol of the operators. The second uses the Voros symbol based on coherent states. After some analysis, we show that they lead to identical physics.

\subsection{The Commutative Case}

\subsubsection{The Positive Map}

The algebra $\mathcal{C}$ in the commutative case is $\mathcal{A}_{0}\left(\mathbb{R}^{2}\right)=C^{\infty}(\mathbb{R} \times \mathbb{R})$, the product being pointwise multiplication, and $*$ being complex conjugation. If $\psi \in \mathcal{C}$, then $\psi\left(x_{0}, x_{1}\right) \in \mathbb{C}$, where $\left(x_{0}, x_{1}\right)$ are coordinates of $\mathbb{R}^{2}$.

There is no distinction now between $\hat{\alpha}^{L}$ and $\hat{\alpha}^{R}: \hat{\alpha}^{L}=\hat{\alpha}^{R}$.

There is actually a family of positive maps $\chi_{t}$ of interest obtained by integrating $\psi$ in $x_{1}$ at "time" $t$ :

$$
\begin{gathered}
\chi_{t}(\psi)=\int d x_{1} \psi\left(t, x_{1}\right), \\
\chi_{t}\left(\psi^{*} \psi\right) \geq 0 .
\end{gathered}
$$

This defines a family of spaces $\mathcal{C}_{t}$ with a positive-definite sesquilinear form (an "inner product") $(., .)_{t}$ :

$$
(\psi, \varphi)_{t}=\int d x_{1} \psi^{*}\left(t, x_{1}\right) \varphi\left(t, x_{1}\right)
$$


(We associate $\chi_{t}$ with $\mathcal{C}$ to get $\mathcal{C}_{t}$.)

\subsubsection{The Null Space $\mathcal{N}_{t}^{0}$}

Every function $\hat{\alpha}$ which vanishes at time $t$ is a two-sided ideal $\mathcal{I}_{t}^{\theta=0}:=\mathcal{I}_{t}^{0}$ of $\mathcal{C}$. As elements of $\mathcal{C}_{t}$, they become null vectors $\mathcal{N}_{t}^{0}$ in the inner product (3.4). (We associate $\chi_{t}$ also to $\mathcal{I}_{t}^{0}$ to get $\mathcal{N}_{t}^{0}$.) Thus as in the GNS construction [17], we can quotient by these vectors and work with $\mathcal{C}_{t} / \mathcal{N}_{t}^{0}$. For elements $\psi+\mathcal{N}_{t}^{0}$ and $\chi+\mathcal{N}_{t}^{0}$ in $\mathcal{C}_{t} / \mathcal{N}_{t}^{0}$, the scalar product is

$$
\left(\psi+\mathcal{N}_{t}^{0}, \chi+\mathcal{N}_{t}^{0}\right)_{t}=(\psi, \chi)_{t}
$$

There are no non-trivial vectors of zero norm now. The completion $\overline{\mathcal{C}_{t} / \mathcal{N}_{t}^{0}}$ of $\mathcal{C}_{t} / \mathcal{N}_{t}^{0}$ in this scalar product gives a Hilbert space $\widehat{\mathcal{H}}_{t}^{0}$. We have also that $\mathcal{C}_{t} / \mathcal{I}_{t}^{0}$ acts on it faithfully, preserving its $*$,

$$
\left(\psi+\mathcal{I}_{t}^{0}\right)^{*}=\psi^{*}+\left(\mathcal{I}_{t}^{0}\right)^{*}=\psi^{*}+\mathcal{I}_{t}^{0}
$$

In the expression above, $S^{*}$ is the set obtained from $S$ by taking the complex conjugate of each element. Hence $\left(\mathcal{I}_{t}^{0}\right)^{*}=\mathcal{I}_{t}^{0}$.

\subsubsection{The Quantum Mechanical Hilbert Space $\mathcal{H}_{t}^{0}$}

The quantum mechanical Hilbert space however is not $\widehat{\mathcal{H}}_{t}^{0}$. It is constructed in a different way, starting from a subspace $\tilde{\mathcal{H}}_{0, t} \subset \mathcal{C}_{t}$ which contains only $\{0\}$ as the null vector:

$$
\tilde{\mathcal{H}}_{0, t} \cap \mathcal{N}_{t}^{0}=\{0\}
$$

(The subscript 0 on $\tilde{\mathcal{H}}_{0, t}$ denotes the value of $\theta$.) Then $\chi_{t}$ is a good scalar product on $\tilde{\mathcal{H}}_{0, t}$ and the quantum mechanical Hilbert space is given by $\mathcal{H}_{t}^{0}=\overline{\tilde{\mathcal{H}}_{0, t}}$, the completion of $\tilde{\mathcal{H}}_{0, t}$.

The subspace $\tilde{\mathcal{H}}_{0, t}$ depends on the Hamiltonian $H$ and is chosen as follows. Suppose first that $H$ is a time-independent Hamiltonian on commutative spacetime, self-adjoint on the standard quantum mechanical Hilbert space $L^{2}(\mathbb{R})$. It acts on $\mathcal{C}_{t}$ and obeys

$$
(\psi, H \chi)_{t}=(H \psi, \chi)_{t}
$$

We now pick the subspace $\tilde{\mathcal{H}}_{0, t}$ of $\mathcal{C}_{t}$ by requiring that vectors in $\mathcal{C}_{t}$ obey the timedependent Schrödinger equation:

$$
\tilde{\mathcal{H}}_{0, t}=\left\{\psi \in \mathcal{C}_{t}:\left(i \partial_{x_{0}}-H\right) \psi\left(x_{0}, x_{1}\right)=0\right\} .
$$

The operator $i \partial_{x_{0}}$ is not "hermitian" on all vectors of $\mathcal{C}_{t}$ :

$$
\left(\psi, i \partial_{x_{0}} \chi\right)_{t} \neq\left(i \partial_{x_{0}} \psi, \chi\right)_{t} \text { for generic } \psi, \chi \in \mathcal{C}_{t}
$$

but on $\tilde{\mathcal{H}}_{0, t}$, it equals $H$ and does fulfill this property:

$$
\left(\psi, i \partial_{x_{0}} \chi\right)_{t}=\left(i \partial_{x_{0}} \psi, \chi\right)_{t} \text { for generic } \psi, \chi \in \tilde{\mathcal{H}}_{0, t} .
$$

Since $\left[i \partial_{x_{0}}, H\right]=0$, both $i \partial_{x_{0}}$ and $H$ leave the subspace $\tilde{\mathcal{H}}_{0, t}$ invariant:

$$
i \partial_{x_{0}} \tilde{\mathcal{H}}_{0, t}=H \tilde{\mathcal{H}}_{0, t} \subseteq \tilde{\mathcal{H}}_{0, t}
$$


We see also that since

$$
\psi\left(x_{0}+\tau, x_{1}\right)=\left(e^{-i \tau\left(i \partial_{x_{0}}\right)} \psi\right)\left(x_{0}, x_{1}\right)=\left(e^{-i \tau H} \psi\right)\left(x_{0}, x_{1}\right),
$$

time evolution preserves the norm of $\psi \in \tilde{\mathcal{H}}_{0, t}$. Therefore if it vanishes at $x_{0}=t$, it vanishes identically and is the zero element of $\tilde{\mathcal{H}}_{0, t}$ : the only null vector in $\tilde{\mathcal{H}}_{0, t}$ is 0 :

$$
\mathcal{N}_{t}^{0} \cap \tilde{\mathcal{H}}_{0, t}=\{0\}
$$

That means that $\chi_{t}$ gives a true scalar product on $\tilde{\mathcal{H}}_{0, t}$. The completion of $\tilde{\mathcal{H}}_{0, t}$ is the quantum Hilbert space $\mathcal{H}_{t}^{0}$.

We can find no convenient inclusion of $\mathcal{H}_{t}^{0}$ in $\widehat{\mathcal{H}}_{t}^{0}$. The reason is that $\mathcal{N}_{t}^{0}$ is not in the kernel of $\left(i \partial_{x_{0}}-H\right)$, only its zero vector is.

Elements of $\tilde{\mathcal{H}}_{0, t}$ are very conventional. Let $\hat{x}_{\mu}$ be coordinate functions $\left(\hat{x}_{\mu}\left(x_{0}, x_{1}\right)=\right.$ $x_{\mu}$ ) so that $i \partial_{x_{0}} \hat{x}_{\mu}=i \delta_{0 \mu}$, and let $\psi_{0}$ be a constant function of $x_{0}$ so that $i \partial_{x_{0}} \psi_{0}=0$. Then

$$
\psi=e^{-i \hat{x}_{0} H} \psi_{0} \in \tilde{\mathcal{H}}_{0, t} .
$$

Under time evolution by amount $\tau, \psi$ becomes

$$
e^{-i \tau H} \psi=e^{-i\left(\hat{x}_{0}+\tau\right) H} \psi_{0} \in \tilde{\mathcal{H}}_{0, t}
$$

The conceptual difference between coordinate time $\hat{x}_{0}$ and amount of time translation $\tau$ is apparent here. As one learns from Doplicher et al. [1], this difference cannot be ignored with spacetime noncommutativity.

As $\psi_{0}$ is constant in $x_{0}$, its values may be written as $\psi_{0}\left(x_{1}\right)$.

\subsubsection{On Observables}

An observable $\hat{K}$ has to respect the Schrödinger constraint and leave $\tilde{\mathcal{H}}_{0, t}$ (and hence $\mathcal{H}_{t}^{0}$ ) invariant. This means that

$$
\left[i \partial_{x_{0}}-H, \hat{K}\right]=0
$$

Let $\hat{L}$ be any operator with no explicit time dependence so that $\hat{L}$ is a function of $\hat{x}_{1}$ and momentum. Then

$$
\hat{K}=e^{-i \hat{x}_{0} H} \hat{L} e^{+i \hat{x}_{0} H}
$$

is an observable. We have also that $\hat{K}$ acts on $\psi$ in a familiar manner:

$$
\hat{K} \psi=\left(\hat{L} \psi_{0}\right) e^{-i \hat{x}_{0} H} .
$$

Under time translation, $\hat{x}_{0}$ in $\hat{K}$ shifts to $\hat{x}_{0}+\tau$ as it should:

$$
e^{-i \tau H} \hat{K} e^{+i \tau H}=e^{-i\left(\hat{x}_{0}+\tau\right) H} \hat{L} e^{+i\left(\hat{x}_{0}+\tau\right) H} .
$$

Response under time-translations is dynamics, it gives time-evolution. Just as in the conventional approach, here and elsewhere we should time-evolve either vector states 
(Schrödinger representation) or observables (Heisenberg representation). One can also formulate the interaction representation.

A final important point is the following. The observables have the expected reality properties. In particular, $\mathcal{C}$ is a $*$-algebra, with star being complex conjugation, denoted here by a bar. So are the functions $\hat{L}$ on $\mathbb{R}^{2}$ which are constant in $x_{0}$, that is, functions of position only. If $\hat{K}$ is its image on $\mathcal{H}_{t}^{0}$, as in (3.18), then $\overline{\hat{L}}$ has image $\hat{K}^{\dagger}$ : we have a *-representation of these functions. Momentum too is a self-adjoint operator on $\mathcal{H}_{t}^{0}$.

\subsubsection{Time-dependent $H$}

Next suppose that $H$ has $\hat{x}_{0}$-dependence:

$$
\left[i \partial_{x_{0}}, H\right] \neq 0 .
$$

This means that $H$ is a function of $\hat{x}_{0}$ and other operators like $\hat{x}_{1}$ and momentum, and we should write for the Hamiltonian $H\left(\hat{x}_{0}, \hat{x}_{1},-i \partial_{x_{1}}\right)$. There is no factor-ordering problem involving $\hat{x}_{0}$ here. We can substitute a real variable $x_{0}$ for $\hat{x}_{0}$ and get the operator $H\left(x_{0}, \hat{x}_{1},-i \partial_{x_{1}}, \ldots\right)$ without ambiguity.

The Schrödinger constraint (3.17) remains intact, but $\psi \in \tilde{\mathcal{H}}_{0, t}$ has a different expression:

$$
\begin{gathered}
\psi=U\left(\hat{x}_{0}, \tau_{I}\right) \psi_{0} \\
U\left(\hat{x}_{0}, \tau_{I}\right)=\left.T \exp \left[-i \int_{\tau_{I}}^{x_{0}} d x_{0}^{\prime} H\left(x_{0}^{\prime}, \hat{x}_{1},-i \partial_{x_{1}}, \ldots\right)\right]\right|_{x_{0}=\hat{x}_{0}} .
\end{gathered}
$$

where $\tau_{I}$ is the initial time at which $\psi=\psi_{0}$ (which depends only on $\hat{x}_{1}$ ), and $T$ is time ordering in $x_{0}^{\prime}$.

Time translation by amount $\tau$ shifts $\hat{x}_{0}$ to $\hat{x}_{0}+\tau$ in $U$ as before: $U\left(\hat{x}_{0}, \tau_{I}\right) \rightarrow$ $U\left(\hat{x}_{0}+\tau, \tau_{I}\right)$. Observables are constructed from $\hat{L}$ using $U$ and have familiar properties.

\subsubsection{Is Time an Observable?}

What we have described above leads to conventional physics. Just as in the latter, here too, $\hat{x}_{0}$ is not an observable as it does not commute with $i \partial_{x_{0}}-H$ :

$$
\left[\hat{x}_{0}, i \partial_{x_{0}}-H\right]=-i \mathbb{I} .
$$

Transformations with $\exp \left(-i \hat{x}_{0} H\right)$ or $U$ does not affect $\hat{x}_{0}$. So we cannot construct an observable therefrom as we $\operatorname{did}$ to get $\hat{K}$ from $\hat{L}$.

\subsubsection{On the Time-dependence of $\mathcal{H}_{t}^{0}$}

In conventional quantum physics, the Hilbert space has no time-dependence, whereas $\mathcal{H}_{t}^{0}$ has a label $t$. This is puzzling.

But the puzzle is easy to resolve: $\mathcal{H}_{t}^{0}$ is independent of $t$. Thus the solutions $\psi$ of the Schrödinger constraint do not depend on $t$ and are elements of every $\mathcal{H}_{t}^{0}$. Their scalar products too are independent of $t$ because of the unitarity of $H$. There is thus only one Hilbert space which we call $\mathcal{H}_{0}(0$ standing for the value of $\theta)$. We also denote $\tilde{\mathcal{H}}_{0, t}$ by $\tilde{\mathcal{H}}_{0}$ henceforth. Further the observables have no explicit $t$-dependence and act on $\mathcal{H}_{0}$ as in standard quantum theory. 


\subsection{The Noncommutative Case}

The above discussion shows that for quantum theory, what we need are: (1) a suitable inner product on $\mathcal{A}_{\theta}\left(\mathbb{R}^{2}\right)$; (2) a Schrödinger constraint on $\mathcal{A}_{\theta}\left(\mathbb{R}^{2}\right)$; and (3) a Hamiltonian $\hat{H}$ and observables which act on the constrained subspace of $\mathcal{A}_{\theta}\left(\mathbb{R}^{2}\right)$. We also require that (1) is compatible with the self-adjointness of $\hat{H}$ and classically real observables.

We now consider these items one by one.

\subsubsection{The Inner Product}

There are several suitable inner products at first sight. But we shall later argue that they are all equivalent.

The first inner product is based on symbol calculus. If $\hat{\alpha} \in \mathcal{A}_{\theta}\left(\mathbb{R}^{2}\right)$, we write it as

$$
\hat{\alpha}=\int d^{2} k \tilde{\alpha}(k) e^{i k_{1} \hat{x}_{1}} e^{i k_{0} \hat{x}_{0}},
$$

and associate the symbol $\alpha_{S}$ with $\hat{\alpha}$ where

$$
\alpha_{S}\left(x_{0}, x_{1}\right)=\int d^{2} k \tilde{\alpha}(k) e^{i k_{1} x_{1}} e^{i k_{0} x_{0}} .
$$

The symbol is a function on $\mathbb{R}^{2}$. It is not the Moyal symbol. For the latter, the exponentials in (3.24) must be written as $\exp \left(i k_{1} \hat{x}_{1}+i k_{0} \hat{x}_{0}\right)$.

Using this symbol, we can define a positive map $S_{t}$ by

$$
S_{t}(\hat{\alpha})=\int d x_{1} \alpha_{S}\left(t, x_{1}\right) .
$$

Properties of $S_{t}$ are similar to $\chi_{t}$. In particular it gives the inner product $(., .)_{t}$, where

$$
(\hat{\alpha}, \hat{\beta})_{S_{t}}=S_{t}\left(\hat{\alpha}^{*} \hat{\beta}\right)=\int d x_{1} \alpha_{S}^{*}\left(t, x_{1}\right) \beta_{S}\left(t, x_{1}\right) \text {. }
$$

This inner product has null vectors $\mathcal{N}_{t}^{\theta}: \hat{\alpha} \in \mathcal{N}_{t}^{\theta}$ if $\alpha_{S}(t,)=$.0 . But we will not consider them further as the physical Hilbert space $\mathcal{H}_{t}^{\theta}$ is not obtained from $\mathcal{A}_{\theta}\left(\mathbb{R}^{2}\right) / \mathcal{N}_{t}^{\theta}$.

A second inner product can be constructed using the Voros map, based on the coherent states associated with (1.1). Let

$$
a=\frac{\hat{x}_{0}+i \hat{x}_{1}}{\sqrt{2 \theta}}, a^{\dagger}=\frac{\hat{x}_{0}-i \hat{x}_{1}}{\sqrt{2 \theta}},\left[a, a^{\dagger}\right]=\mathbb{I},
$$

and introduce the coherent states

$$
\left|z=x_{0}+i x_{1}\right\rangle=e^{\frac{1}{\sqrt{2 \theta}}\left(z a^{\dagger}-\bar{z} a\right)}|0\rangle .
$$

The Voros or coherent state symbol of an operator $\hat{\alpha} \in \mathcal{A}_{\theta}\left(\mathbb{R}^{2}\right)$ is the function $\alpha_{V}$ on $\mathbb{R}^{2}$ where

$$
\alpha_{V}\left(x_{0}, x_{1}\right)=\langle z|\hat{\alpha}| z\rangle
$$


The positive map $V_{t}$ is then defined by

$$
V_{t}(\hat{\alpha})=\int d x_{1} \alpha_{V}\left(t, x_{1}\right)
$$

As the symbol of a positive operator $\hat{\alpha}^{*} \hat{\alpha}$ is a non-negative function, the positivity of the map $V_{t}$ is manifest from (3.31). There are also no nontrivial null vectors in the scalar product

$$
(\hat{\alpha}, \hat{\beta})_{V_{t}}=V_{t}\left(\hat{\alpha}^{*} \hat{\beta}\right)
$$

as one can show. But that result is not important for what follows as the Hilbert space is obtained only after constraining the vector states by the noncommutative Schrödinger equation.

\subsubsection{The Schrödinger Constraint}

The noncommutative analogue " $i \frac{\partial}{\partial x_{0}}$ " of the corresponding commutative operator is

$$
i \frac{\partial}{\partial x_{0}} \equiv \hat{P}_{0}=-\frac{1}{\theta} \operatorname{ad} \hat{x}_{1}
$$

since

$$
-\frac{1}{\theta} \operatorname{ad} \hat{x}_{1} \hat{x}_{\lambda}=i \delta_{\lambda 0} \mathbb{I}
$$

If the Hamiltonian $\hat{H}$ is time-independent,

$$
\left[i \partial_{x_{0}}, \hat{H}\right]=0
$$

it depends on the momentum $\hat{P}_{1}$ in $(2.5)$ and $\hat{x}_{1}^{L}$, and we can write it as

$$
\hat{H}=\hat{H}\left(\hat{x}_{1}^{L}, \hat{P}_{1}\right)
$$

It can depend on $\hat{x}_{1}^{R}$ as well if we rely just on (3.35). But since $\hat{x}_{1}^{R}=-\operatorname{ad} \hat{x}_{1}+\hat{x}_{1}^{L}$, that means $\hat{H}$ has dependence also on $i \partial_{x_{0}}$ and we can write

$$
\hat{H}=\hat{H}\left(\hat{x}_{1}^{L}, \hat{P}_{1}, i \partial_{x_{0}}\right)
$$

This generalisation however seems unwarranted: there is never such dependence of $H$ on $i \partial_{x_{0}}$ for $\theta=0$, and we will generally obtain $\hat{H}$ from $H$ in a manner that does not induce this dependence.

If $\hat{H}$ has time-dependence and (3.35) is not correct, it will have $\hat{x}_{0}^{L}, \hat{x}_{0}^{R}$ or both in its arguments. But $\hat{x}_{0}^{L}=\theta \hat{P}_{1}+\hat{x}_{1}^{R}$, so in the time-dependent case we write

$$
\hat{H}=\hat{H}\left(\hat{x}_{0}^{R}, \hat{x}_{1}^{L}, \hat{P}_{1}\right),
$$

ignoring a possible $i \partial_{x_{0}}$ dependence for reasons above.

The family of vector states constrained by the Schrödinger equation is

$$
\tilde{\mathcal{H}}_{\theta}=\left\{\hat{\psi} \in \mathcal{A}_{\theta}\left(\mathbb{R}^{2}\right):\left(i \partial_{x_{0}}-\hat{H}\right) \hat{\psi}=0\right\},
$$


where arguments of $\hat{H}$ can be appropriately inserted.

The solutions of (3.39) are easy to come by. For the time-independent case,

$$
\hat{\psi} \in \tilde{\mathcal{H}}_{\theta} \Longrightarrow \hat{\psi}=e^{-i\left(\hat{x}_{0}^{R}-\tau_{I}\right) \hat{H}\left(\hat{P}_{1}, \hat{x}_{1}^{L}\right)} \hat{\chi}\left(\hat{x}_{1}\right) .
$$

The product $\hat{x}_{0}^{R} \hat{H}$ has no ordering problem since $\left[\hat{x}_{0}^{R}, \hat{H}\left(\hat{x}_{1}^{L}, \hat{P}_{1}\right)\right]=0$. Also $\tau_{I}$ is the initial time when $\hat{\psi}=\hat{\chi}$. Since $\hat{x}_{0}^{R}, \hat{x}_{1}^{L}$ occur in the first factor, we should read the R.H.S. as the exponential acting on the algebra element $\hat{\chi}\left(\hat{x}_{1}\right)$.

Suppose next that $\hat{H}$ depends on $\hat{x}_{0}^{R}$ as in (3.38). As $\hat{x}_{0}^{R}$ commutes with $\hat{P}_{1}$ and $\hat{x}_{1}^{L}$, we can easily generalise the formula 3.40 to write

$$
\begin{gathered}
\hat{\psi} \in \tilde{\mathcal{H}}_{\theta} \Longrightarrow \hat{\psi}=U\left(\hat{x}_{0}^{R}, \tau_{I}\right) \hat{\chi}\left(\hat{x}_{1}\right), \\
U\left(\hat{x}_{0}^{R}, \tau_{I}\right)=\left.T \exp \left[-i\left(\int_{\tau_{I}}^{x_{0}} d \tau \hat{H}\left(\tau, \hat{x}_{1}^{L}, \hat{P}_{1}\right)\right)\right]\right|_{x_{0}=\hat{x}_{0}^{R}} .
\end{gathered}
$$

Just as in (3.40), the dependence of $U$ on $\hat{x}_{0}^{R}$ and $\tau_{I}$ has been displayed, while $\tau_{I}$ is the initial time when $\hat{\psi}=\hat{\chi}$.

Time translation by amount $\tau$ shifts $\hat{x}_{0}^{R}$ to $\hat{x}_{0}^{R}+\tau$ in both (3.40) and (3.41).

An alternative useful form for $\hat{\psi}$ in (3.41) is

$$
\begin{gathered}
\hat{\psi}=V\left(\hat{x}_{0}^{R},-\infty\right) \hat{\chi}\left(\hat{x}_{1}\right), \\
V\left(\hat{x}_{0}^{R},-\infty\right)=T \exp \left[-i \int_{-\infty}^{0} d \tau \hat{H}\left(\hat{x}_{0}^{R}+\tau, \hat{x}_{1}^{L}, \hat{P}_{1}\right)\right],
\end{gathered}
$$

where the integral can be defined at the lower limit using the usual adiabatic cut-off.

The Hilbert spaces $\mathcal{H}_{\theta}^{S}$ and $\mathcal{H}_{\theta}^{V}$ based on scalar products $(.,)_{S}$ and $(., .)_{V}$ are obtained from $\tilde{\mathcal{H}}_{\theta}$ by completion. Our basic assumption is that $\hat{H}$ is self-adjoint in the chosen scalar product. Then as before, the resultant Hilbert space $\mathcal{H}_{\theta}^{S}$ or $\mathcal{H}_{\theta}^{V}$ has no dependence on $t$.

Assuming that

$$
\hat{H}=\frac{\hat{P}_{1}^{2}}{2 m}+V\left(\hat{x}_{1}\right)
$$

is a self-adjoint Hamiltonian for $\theta=0$, then we note that its $\theta \neq 0$ version

$$
\hat{H}=\frac{\hat{P}_{1}^{2}}{2 m}+V\left(\hat{x}_{1}^{L}\right)
$$

is self-adjoint on both $\mathcal{H}_{\theta}^{S}$ and $\mathcal{H}_{\theta}^{V}$.

If $\hat{H}\left(\hat{x}_{0}, \hat{x}_{1}, \hat{P}_{1}\right)$ is time-dependent for $\theta=0$, we can form its $\theta \neq 0$ version

$$
\hat{H}\left(\hat{x}_{0}^{L}, \hat{x}_{1}^{L}, \hat{P}_{1}\right)=\hat{H}\left(-\theta \hat{P}_{1}+\hat{x}_{0}^{R}, \hat{x}_{1}^{L}, \hat{P}_{1}\right)
$$

As $\hat{x}_{0}^{L}$ and $\hat{P}_{1}$ do not commute with $\hat{x}_{1}^{L}$, we should check this $\hat{H}$ for factor-ordering problems. But for this potential trouble, $\hat{H}$ is self-adjoint if $H$ is. 


\subsubsection{Remarks on Time for $\theta \neq 0$}

In the passage from $H$ to $\hat{H}$, there is an apparent ambiguity. Above we replaced $x_{0}$ by $\hat{x}_{0}^{L}$, but we may be tempted to replace $x_{0}$ by $\hat{x}_{0}^{R}$. In that case the passage to $\theta \neq 0$ will involve no factor-ordering problem as $\hat{x}_{0}^{R}$ commutes with $\hat{x}_{1}^{L}$ and $\hat{P}_{1}$. At the same time, $\theta$-dependent terms in $\hat{H}$ disappear.

But it is incorrect to replace $x_{0}$ by $\hat{x}_{0}^{R}$ and at the same time $x_{1}$ by $\hat{x}_{1}^{L}$. Time and space should fulfill the relation (1.1) when $\theta$ becomes nonzero whereas $\hat{x}_{0}^{R}$ and $\hat{x}_{1}^{L}$ commute.

Note that $\hat{x}_{0}^{L, R}$ do not preserve the Schrödinger constraint so that there is no time operator for $\theta \neq 0$ as well.

\subsubsection{Time-dependence for $\theta=0 \Longrightarrow$ Spatial nonlocality for $\theta \neq 0$}

We noted above that $\hat{x}_{0}^{L}=-\theta \hat{P}_{1}+\hat{x}_{0}^{R}$ and that $\hat{x}_{0}^{R}$ behaves much like the $\theta=0$ time $x_{0}$. Thus if $H$ has time-dependence, its effect on $\hat{H}$ is to induce new momentum-dependent terms. The $x_{0}$-dependence in $H$ need not to be polynomial so that in $\hat{H}$ they induce nonpolynomial interactions in momentum, that is, instantaneous spatially nonlocal ("acausal") interactions.

\subsubsection{Observables}

We can construct observables as in (3.18) or its version for time-dependent Hamiltonians. No complications are encountered.

\subsubsection{The Scalar Products $(., .)_{S}$ and $(., .)_{V}$}

We now explore the relation between the different scalar products. We assume (as is often the case) that $\hat{H}$ is self-adjoint for both. As the scalar products do not have time dependence, we have dropped their time-subscripts.

It is enough to consider time-independent $\hat{H}$. Let $\hat{\psi}_{n}$ be its eigenstates,

$$
\hat{H} \hat{\psi}_{n}=E_{n} \hat{\psi}_{n},
$$

and assume in the first instance that eigenvalues are non-degenerate:

$$
E_{n} \neq E_{m} \text { if } n \neq m \text {. }
$$

For simplicity, the eigenvalues are taken to be discrete throughout this discussion. Then since $\hat{H}$ is self-adjoint in either scalar product,

$$
\left(\hat{\psi}_{m}, \hat{\psi}_{n}\right)_{S}=s_{n} \delta_{m n},\left(\hat{\psi}_{m}, \hat{\psi}_{n}\right)_{V}=v_{n} \delta_{m n}, s_{n}, v_{n}>0
$$

Thus an isometry from the $S$-Hilbert space $\mathcal{H}_{\theta}^{S}$ to the $V$-Hilbert space $\mathcal{H}_{\theta}^{V}$ (based on the scalar products $(., .)_{S}$ and $(., .)_{V}$ respectively) is

$$
\mathcal{H}_{\theta}^{S} \ni \frac{1}{\sqrt{s_{n}}} \hat{\psi}_{n} \rightarrow \frac{1}{\sqrt{v_{n}}} \hat{\psi}_{n} \in \mathcal{H}_{\theta}^{V}
$$


If $\hat{K}_{S}$ is an observable in the $S$-Hilbert space with matrix $k^{S}$ in the basis $\left\{\left(1 / \sqrt{s_{n}}\right) \hat{\psi}_{n}\right\}$,

$$
\hat{K}_{S} \frac{1}{\sqrt{s_{n}}} \hat{\psi}_{n}=\frac{1}{\sqrt{s_{m}}} \hat{\psi}_{m} k_{m n}^{S}, k_{m n}^{S} \in \mathbb{C}
$$

to it we can associate the operator $\hat{K}_{V}$ on the $V$-Hilbert space defined by

$$
\hat{K}_{V} \frac{1}{\sqrt{v_{n}}} \hat{\psi}_{n}=\frac{1}{\sqrt{v_{m}}} \hat{\psi}_{m} k_{m n}^{S}
$$

Then

$$
\left(\frac{1}{\sqrt{s_{m}}} \hat{\psi}_{m}, \hat{K}_{S} \frac{1}{\sqrt{s_{n}}} \hat{\psi}_{n}\right)_{S}=\left(\frac{1}{\sqrt{v_{m}}} \hat{\psi}_{m}, \hat{K}_{V} \frac{1}{\sqrt{v_{n}}} \hat{\psi}_{n}\right)_{V}
$$

and physics in the two spaces become identical.

If $\hat{H}$ has degeneracies, we can introduce a degeneracy index $r$ and write

$$
\begin{gathered}
\hat{H} \hat{\psi}_{n}^{(r)}=E_{n} \hat{\psi}_{n}^{(r)}, E_{n} \neq E_{m} \text { if } n \neq m \\
\left(\hat{\psi}_{m}^{(r)}, \hat{\psi}_{n}^{(s)}\right)_{S}=s_{m} \delta_{r s} \delta_{m n}, s_{m}>0
\end{gathered}
$$

Then

$$
\left(\hat{\psi}_{m}^{(r)}, \hat{\psi}_{n}^{(s)}\right)_{V}=\delta_{m n} W_{r s}(m)
$$

where $W(m)$ is a positive matrix with a positive invertible square root $W(m)^{1 / 2}$. An isometry from $\mathcal{H}_{\theta}^{S}$ to $\mathcal{H}_{\theta}^{V}$ is thus

$$
\mathcal{H}_{\theta}^{S} \ni \frac{1}{\sqrt{s_{n}}} \hat{\psi}_{n}^{(r)} \rightarrow \hat{\psi}_{n}^{(s)} W_{s r}(n)^{-1 / 2} \in \mathcal{H}_{\theta}^{V}
$$

as is shown using $\left[W(n)^{-1 / 2}\right]^{\dagger}=W(n)^{-1 / 2}$.

Following 3.51) and (3.52), we can also map an observable $\hat{K}_{S}$ in $\mathcal{H}_{\theta}^{S}$ to its equivalent on $\mathcal{H}_{\theta}^{V}$. Write

$$
\hat{K}_{S} \frac{1}{\sqrt{s_{n}}} \hat{\psi}_{n}^{(r)}=\frac{1}{\sqrt{s_{n^{\prime}}}} \hat{\psi}_{n^{\prime}}^{\left(r^{\prime}\right)} k_{n^{\prime} r^{\prime}, n r}^{S}
$$

Then

$$
\hat{K}_{V} \hat{\psi}_{n}^{(s)} W_{s r}(n)^{-1 / 2}=\hat{\psi}_{n^{\prime}}^{(s)} W_{s r^{\prime}}\left(n^{\prime}\right)^{-1 / 2} k_{n^{\prime} r^{\prime}, n r}^{S} .
$$

Similar results are correct for time-dependent $\hat{H}$ and for any scalar product compatible with the self-adjointness of $\hat{H}$.

We note that equivalent observables as elements of the algebra generally differ for such differing scalar products. One universal exception is the Hamiltonian when it is timeindependent.

\section{Examples}

For definiteness, we work hereafter with $\mathcal{H}_{\theta}^{S}$. 


\subsection{Plane Waves}

Let

$$
\hat{H}_{0}=\frac{\hat{P}_{1}^{2}}{2 m}
$$

be the free Hamiltonian. Its eigenstates are

$$
\hat{\psi}_{k}=e^{i k \hat{x}_{1}} e^{-i \omega(k) \hat{x}_{0}}, \omega(k)=\frac{k^{2}}{2 m}, k \in \mathbb{R} .
$$

The eigenvalues are $k^{2} / 2 m$ :

$$
\hat{H}_{0} \hat{\psi}_{k}=\left(\hat{H}_{0} e^{i k \hat{x}_{1}}\right) e^{-i \omega(k) \hat{x}_{0}}=\omega(k) \hat{\psi}_{k} .
$$

The second factor in $\hat{\psi}_{k}$ is dictated by the Schrödinger constraint:

$$
\hat{P}_{0} \hat{\psi}_{k}=e^{i k \hat{x}_{1}} \hat{P}_{0} e^{-i \omega(k) \hat{x}_{0}}=\omega(k) \hat{\psi}_{k} \Longrightarrow\left(\hat{P}_{0}-\hat{H}\right) \hat{\psi}_{k}=0 .
$$

The spectrum of $\hat{H}_{0}$ is completely conventional while the noncommutative plane waves too resemble the ordinary plane waves. But phenomena like beats and interference show new features 18.

The coincidence of spectra of the free Hamiltonians in commutative and noncommutative cases is an illustration of a more general result which we now establish.

\subsection{A Spectral Map}

For $\theta=0$ consider the Hamiltonian

$$
H=-\frac{1}{2 m} \frac{\partial^{2}}{\partial x_{1}^{2}}+V\left(\hat{x}_{1}\right)
$$

with eigenstates $\psi_{E}$ fulfilling the Schrödinger constraint:

$$
\begin{gathered}
\psi_{E}\left(\hat{x}_{0}, \hat{x}_{1}\right)=\varphi_{E}\left(\hat{x}_{1}\right) e^{-i E \hat{x}_{0}}, \\
H \varphi_{E}=E \varphi_{E} .
\end{gathered}
$$

The Hamiltonian $\hat{H}$ associated to $H$ for $\theta \neq 0$ is

$$
\hat{H}=\frac{\hat{P}_{1}^{2}}{2 m}+V\left(\hat{x}_{1}\right) .
$$

Then $\hat{H}$ has exactly the same spectrum as $H$ while its eigenstates $\hat{\psi}_{E}$ are obtained from $\psi_{E}$ just by regarding $\hat{x}_{0}$ and $\hat{x}_{1}$ as fulfilling (1.1):

$$
\begin{gathered}
\hat{\psi}_{E}=\varphi_{E}\left(\hat{x}_{1}\right) e^{-i E \hat{x}_{0}}, \\
\hat{H} \varphi_{E}\left(\hat{x}_{1}\right)=E \varphi_{E}\left(\hat{x}_{1}\right) .
\end{gathered}
$$

The proof of (4.10) follows from (4.8) as it involves no feature associated with spacetime noncommutativity. Since

$$
\hat{P}_{0} \hat{\psi}_{E}=\varphi_{E}\left(\hat{x}_{1}\right) \hat{P}_{0} e^{-i E \hat{x}_{0}}=E \hat{\psi}_{E}
$$

we see that $\hat{\psi}_{E}$ fulfills the Schrödinger constraint as well.

When the spatial slice for a commutative spacetime $\mathbb{R}^{d}$ is of dimension two or larger, one can introduce space-space noncommutativity as well. That would change the noncommutative Hamiltonian. The spectral map may not then exist. 


\section{Conserved Current}

The existence of a current $j_{\lambda}$ which fulfills the continuity equation has a particular importance when $\theta=0$. It is this current which after second quantization couples to electromagnetism [19].

There is such a conserved current also for $\theta \neq 0$. It follows in the usual way from (3.39) and its $*$ :

$$
\left(\hat{P}_{0} \hat{\psi}\right)^{*}-\hat{\psi}^{*} \hat{H}=-\hat{P}_{0} \hat{\psi}^{*}-\hat{\psi}^{*} \hat{H}=0 .
$$

Here we assumed that $\hat{V}^{*}=\hat{V}$.

Multiplying the Schrödinger constraint in (3.39) on left by $\hat{\psi}^{*}$ and (5.1) on right by $\hat{\psi}$ and subtracting,

$$
\hat{P}_{0}\left(\hat{\psi}^{*} \hat{\psi}\right)=\hat{\psi}^{*}\left(\frac{\hat{P}_{1}^{2}}{2 m} \hat{\psi}\right)-\left(\frac{\hat{P}_{1}^{2}}{2 m} \hat{\psi}^{*}\right) \hat{\psi}=\frac{\hat{P}_{1}}{2 m}\left[\hat{\psi}^{*}\left(\hat{P}_{1} \hat{\psi}\right)-\left(\hat{P}_{1} \hat{\psi}^{*}\right) \hat{\psi}\right] .
$$

With

$$
\hat{\rho}=\hat{\psi}^{*} \hat{\psi}, \hat{j}=\frac{1}{2 m}\left[\hat{\psi}^{*}\left(\hat{P}_{1} \hat{\psi}\right)-\left(\hat{P}_{1} \hat{\psi}^{*}\right) \hat{\psi}\right]
$$

as the noncommutative charge and current densities, (5.2) can be interpreted as the noncommutative continuity equation.

\section{Towards Quantum Field Theory}

Perturbative quantum field theories (qft's) based on algebras like (1.1) have been treated with depth by Doplicher et al. [1]. We can also see how to do perturbative qft's, our approach can be inferred from the work of Doplicher et al.

In the interaction representation, an operator $U_{I}$ determines the $S$-matrix. It is in turn determined by the interaction Hamiltonian $\hat{H}_{I}$. The latter is based on "free fields" which are solutions of the Klein-Gordon equation (We assume zero spin for simplicity). Examples of $\hat{H}_{I}$ can be based on interaction Hamiltonians $H_{I}$ such as $\lambda \int d x_{1} \Phi\left(x_{0}, x_{1}\right)^{4}$ (with $\Phi^{\dagger}=\Phi$ being a free field) for $\theta=0$. For this particular $H_{I}, \hat{H}_{I}$ can be something like $\lambda S_{x_{0}}\left[\hat{\Phi}\left(\hat{x}_{0}, \hat{x}_{1}\right)^{4}\right]$ (cf. $(3.26)$ ), where $\hat{\Phi}$ is the self-adjoint free field for $\theta \neq 0$. We make this expression more precise below.

We require of $\hat{\Phi}$ that it is a solution of the massive Klein-Gordon equation:

$$
\left(\operatorname{ad} \hat{P}_{0}^{2}-\operatorname{ad} \hat{P}_{1}^{2}+\mu^{2}\right) \hat{\Phi}=0 .
$$

The plane wave solutions of $(6.1)$ are

$$
\hat{\phi}_{k}=e^{i k \hat{x}_{1}} e^{-i \omega(k) \hat{x}_{0}}, \omega(k)^{2}-k^{2}=\mu^{2} .
$$

So for $\hat{\Phi}$, we write 12

$$
\hat{\Phi}=\int \frac{d k}{2 \omega(k)}\left[a_{k} \hat{\phi}_{k}+a_{k}^{\dagger} \hat{\phi}_{k}^{\dagger}\right]
$$


where $a_{k}$ and $a_{k}^{\dagger}$ commute with $\hat{x}_{\mu}$ and define harmonic oscillators: $\left[a_{k}, a_{k}^{\dagger}\right]=2 \omega(k) \delta(k-$ $\left.k^{\prime}\right)$.

The expression (6.3) is the "free" field "coinciding with the Heisenberg field initially". After time translation by amount $\tau$ using the free Schrödinger Hamiltonian

$$
\hat{H}_{0}=\int \frac{d k}{2 \omega(k)} a_{k}^{\dagger} a_{k}
$$

it becomes

$$
U_{0}(\tau)(\hat{\Phi})=e^{i \tau \hat{H}_{0}} \hat{\Phi} e^{-i \tau \hat{H}_{0}}
$$

The interaction Hamiltonian is accordingly

$$
\hat{H}_{I}\left(x_{0}\right)=\lambda: S_{x_{0}}\left(U_{0}(\tau)(\hat{\Phi})^{4}\right):=\lambda: S_{x_{0}+\tau}\left(\hat{\Phi}^{4}\right):, \lambda>0
$$

where : : denotes the normal ordering of $a_{k}$ and $a_{k}^{\dagger}$.

The $S$-matrix $S$ can be worked out as usual:

$$
S=T \exp \left[-i \int_{-\infty}^{+\infty} d \tau \lambda: S_{\tau}\left(\hat{\Phi}^{4}\right):\right] .
$$

It is important to recognise, as is clear from Doplicher et al. [1], that time-ordering is with respect to the time-translation parameter $\tau$ and not the spectrum of the operator $\hat{x}_{0}^{L}$. Its perturbation series can be developed since we understand the relevant properties of $\hat{\Phi}$.

Scattering amplitudes can be calculated from (6.7). There is no obvious reason why they are not compatible with perturbative unitarity [20].

\section{Acknowledgments}

We have benefitted from discussions with K. S. Gupta, P. Presnajder, A. Pinzul, A. Stern and S. Vaidya. Kumar Gupta and Peter Presnajder were particularly helpful with detailed comments. We also thank W. Bietenholz for the reference to Yang in [-4] and R. Jackiw for reminding us of the work in [10, 11]. This work was partially supported by DOE under

contract number DE-FG02-85ER40231, by NSF under contract number INT9908763 and by FAPESP, Brazil.

\section{References}

[1] S. Doplicher, K. Fredenhagen and J. Roberts, Phys. Lett. B 331, 39 (1994); S. Doplicher, K. Fredenhagen and J. Roberts, Comm. Math. Phys. 172, 187 (1995) hep-th/0303037.

[2] R. Jackiw, Nucl.Phys.Proc.Suppl. 108, 30 (2002) hep-th/0110057; Letter of Heisenberg to Peierls (1930), Wolfgang Pauli, Scientific Correspondence, Vol. II, p.15, Ed. Karl von Meyenn, Springer-Verlag, 1985; Letter of Pauli to Oppenheimer (1946), Wolfgang Pauli, Scientific Correspondence, Vol. III, p.380, Ed. Karl von Meyenn, Springer-Verlag, 1993.

[3] H. Snyder, Phys. Rev. 71, 38 (1947).

[4] C. N. Yang, Phys. Rev. 72, 874 (1947). 
[5] J. Madore, An Introduction to Noncommutative Differential Geometry and its Physical Applications. 2nd. ed, Cambridge, Cambridge Univ. Press, 1999.

[6] A. Kempf, G. Mangano, R. B. Mann, Phys.Rev. D52, 1108 (1995) hep-th/9412167. For related work, see D. V. Ahluwalia, Phys. Lett. B 339, 301 (1994) gr-qc/9308007 and D. V. Ahluwalia, Phys. Lett. A 275, 31 (2000) gr-qc/0002005.

[7] Fedele Lizzi, Gianpiero Mangano, Gennaro Miele and Marco Peloso, J. High Energy Phys. 0206, 049 (2002) hep-th/0203099.

[8] But there are exceptions, currently under study by A. P. Balachandran and A. Pinzul. They come about because time ceases to be an operator on the physical Hilbert space.

[9] M.M. Sheikh-Jabbari, Phys. Rev. Lett. 84, 5265 (2000) hep-th/0001167.

[10] L. Susskind, The Quantum Hall Fluid and Non-Commutative Chern Simons Theory hep-th/0101029.

[11] R. Jackiw, S.-Y. Pi, A.P. Polychronakos, Annals Phys. 301, 157 (2002) hep-th/0206014; B. Bistrovic, R. Jackiw, H. Li, V.P. Nair, S.-Y. Pi, Phys.Rev. D 67025013 (2003) hep-th/0210143.

[12] M. Chaichian, A. Demichev, P. Presnajder, A. Tureanu, Eur. Phys. J. C 20, 767 (2001) hep-th/0007156.

[13] S. Doplicher, private communication.

[14] R. Brunetti and K. Fredenhagen, Phys. Rev. A 66, 044101 (2002) quant-ph/0103144.

[15] Streater, R.F., Wightman, A.S., PCT, spin and statistics, and all that, New York, Benjamin, 1964; Bogoliubov, N.N., Logunov, A.A., Todorov, I.T., Oksak, A.I, General principles of quantum field theory, Dordrecht, Kluwer Academic Publishers, 1990.

[16] Proceedings of the Conference on Spin-Statistics Connection and Commutation Relations, Capri, 2000, Eds. R. C. Hilborn and G. M. Tino, AIP Conference Proceedings, vol.545, 2000.

[17] R. Haag, Local Quantum Physics. Field, Particles, Algebras. 2nd. ed, Berlim, Springer, 1996.

[18] A. P. Balachandran, K. S. Gupta, S. Kurkcuoglu, in preparation.

[19] A. Pinzul, A. Stern, Space-Time Noncommutativity from Particle Mechanics hep-th/0402220.

[20] However see in this connection D. Bak, S. Kim, JHEP 0405053 (2004) hep-th/0310123. 